\title{
Two novel pathogenic variants of L1CAM gene in two fetuses with isolated $X$-linked hydrocephaly: A case report
}

\author{
BOBO XIE ${ }^{1,2}$, JINGSI LUO ${ }^{1,2}$, YAQIN LEI ${ }^{1,2}$, QI YANG ${ }^{1,2}$, MENGTING LI ${ }^{1,2}$, SHANG YI $^{1,2}$, SHIYU LUO $^{1,2}$, \\ JIN WANG ${ }^{1,2}$, ZAILONG QIN $^{1,2}$, ZUOJIAN YANG $^{3}$, HONGWEI WEI $^{4}$ and XIN FAN ${ }^{1,2}$ \\ ${ }^{1}$ Genetic and Metabolic Central Laboratory; ${ }^{2}$ Guangxi Center for Birth Defects Research and Prevention; \\ Departments of ${ }^{3}$ Ultrasound, and ${ }^{4}$ Obstetrics and Gynecology, Guangxi Maternal and Child Health Hospital, \\ Nanning, Guangxi 530023, P.R. China
}

Received January 24, 2018; Accepted October 2, 2018

DOI: $10.3892 / \mathrm{mmr} .2018 .9583$

\begin{abstract}
Hydrocephalus due to aqueductal stenosis (HSAS; Online Mendelian Inheritance in Man \#307000) is a rare $\mathrm{X}$-linked, recessively-inherited disease characterized by severe hydrocephaly and occasionally adducted thumbs, in addition to intellectual disability and spasticity in surviving individuals. The present study described two fetuses with severely enlarged ventricles of the brain. The clinical diagnosis of HSAS was made on the basis of family history and sonographic findings. Molecular testing of the L1 cell adhesion molecule (L1CAM) gene revealed two novel hemizygous $L 1 C A M$ variants, c.998C >T(p.Pro333Leu) and c.2362G>T(p.Val788Phe). The variants affect the highly conserved amino acids which are located in the key domains of the protein (the fourth Ig domain and second FnIII domain, respectively). The two variants were predicted to be 'disease causing' by a number of prediction tools, and have been classified as likely pathogenic following the American College of Medical Genetics and Genomics/Association for Molecular Pathology guidelines. The present study highlights the importance of combining family history, prenatal ultrasonography and molecular testing in the diagnosis of HSAS. The novel variants expand the mutational spectrum of LICAM gene in the Chinese population, and could be used in genetic counseling, carrier testing of female relatives, and prenatal, as well as preimplantation genetic diagnosis.
\end{abstract}

Correspondence to: Professor Xin Fan, Genetic and Metabolic Central Laboratory, Guangxi Maternal and Child Health Hospital, 59 Xiangzhu Road, Nanning, Guangxi 530023, P.R. China E-mail: fanxin602@163.com

Key words: L1 cell adhesion molecule, novel variant, sonographic examination, fetus, X-linked hydrocephaly

\section{Introduction}

X-linked hydrocephalus due to aqueductal stenosis [HSAS, Online Mendelian Inheritance in Man (OMIM) \#307000], affecting $\sim 1$ in 30,000 live male births (1), is the most common of the inherited form of hydrocephalus. It is caused by mutations in the L1 cell adhesion molecule (LICAM) gene; $>280$ different mutations have been reported, and $\sim 50 \%$ of them are missense mutations (2). As the majority of the mutations occur only in one family, they are considered to be private mutations. Genotype-phenotype correlation has been described and L1CAM mutations have been divided into four classes: i) Frameshift or nonsense mutations (Class I) lying in the extracellular L1 protein resulting in loss of L1 protein function and severe phenotypes; ii) missense mutations (Class II) in the extracellular of $\mathrm{L} 1$ resulting in partial conservation of L1 protein function and causing mild or severe phenotypes; iii) mutations in the cytoplasmic domain of L1 (Class III) which tend to affect signal transduction and cause mild phenotypes; and iv) extracellular mutations (Class IV) associated with the aberrant splicing of pre-mRNA, although no corresponding phenotypes have been reported (3).

Apart from HSAS, mutations in LICAM cause three other associated neurological disorders, known collectively as L1 syndrome: Mental retardation, aphasia, shuffling gait, adducted thumbs (MASA) syndrome (OMIM no: 303350); corpus callosum hypoplasia, retardation, adducted thumbs, spastic paraplegia, hydrocephalus (CRASH) syndrome (OMIM no: 303350) and X-linked partial agenesis of corpus callosum (OMIM no: 304100) (4). These allelic disorders are different presentations of L1CAM mutations.

At present, mutations in $L 1 C A M$ in the Chinese population have rarely been reported $(5,6)$. In the present study, two fetuses with isolated hydrocephaly from two Chinese families with a history of X-linked hydrocephalus and the molecular findings were reported.

\section{Case report}

Fetus 1, family 1. A 20-year-old woman, gravida 2 para 0, was referred to the Guangxi Maternal and Child Health Hospital (Nanning, China) on March 14, 2017 at 24 weeks of gestation 
due to sonographic detection of fetal hydrocephaly. The family history was notable; a son with congenital hydrocephaly had been terminated at 28 weeks of gestation (Fig. 1A). A 3D ultrasound examination confirmed a fetus with bilateral dilatation of the cerebral posterior horns [2.54 cm (left), $2.16 \mathrm{~cm}$ (right)], consistent with hydrocephaly (Fig. 1B).

Fetus 2, family 2. A 27-year-old healthy woman, gravida 4 para 2, was referred to the Guangxi Maternal and Child Health Hospital (Nanning, China) on April 8, 2017 at 30 weeks of gestation due to sonographic detection of fetal hydrocephaly. The pregnancy progressed uneventfully, without exposure to any known teratogens. The family history was notable; a son had been terminated due to severe hydrocephalus. There were two healthy daughters (Fig. 1C). A standard second trimester ultrasound screening, performed at the 28th week of gestation, revealed that the width of the left lateral brain ventricle was $3.8 \mathrm{~cm}$ and the right $4.0 \mathrm{~cm}$, consistent with severe hydrocephaly (Fig. 1D). No other fetal structural abnormalities were identified.

A total of 100 healthy individuals of Chinese ethnicity (50 males and 50 females, aged 1-60 years) were recruited at the Guangxi Maternal and Child Health Hospital between May 2017 to December 2017. With informed consent from all participants and approval from the Guangxi Maternal and Child Health Hospital Medical Ethics Committee [approval no. (2017) Lun Han Shen (3-10)], cordocentesis was performed for the two fetuses, and peripheral blood was obtained from the fetuses' family members and the healthy individuals.

Molecular analysis. Genomic DNA was extracted from the cord blood of the fetuses and the peripheral blood of both parents and family members according to the standard protocols of the QIAamp DNA Blood Mini kit (Qiagen $\mathrm{GmbH}$, Hilden, Germany). Fetal DNA was analyzed for $L 1 C A M$ mutations by Sanger sequencing. The primers of the 28 exons with coding region and splicing junctions of $L 1 C A M$ used for amplification and sequencing were designed by Primer3 web (http://primer3.ut.ee; version 4.0.0). The primer sequences are shown in Table I. Polymerase chain reaction was conducted

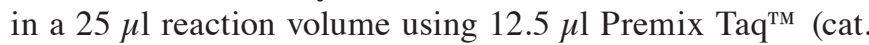
no. RR901A; Takara Biotechnology Co., Ltd., Dalian, China), $9.5 \mu \mathrm{l}$ distilled water, $200 \mathrm{nmol} / \mathrm{l}$ primer and $100 \mathrm{ng} / \mu 1 \mathrm{DNA}$. The thermocycling conditions were as follows: Initial denaturation for $5 \mathrm{~min}$ at $95^{\circ} \mathrm{C}$, followed by 35 cycles of $30 \mathrm{sec}$ at $95^{\circ} \mathrm{C}, 30 \mathrm{sec}$ at $60^{\circ} \mathrm{C}, 60$ or $90 \mathrm{sec}$ at $72^{\circ} \mathrm{C}$, and final extension for $7 \mathrm{~min}$ at $72^{\circ} \mathrm{C}$. DNA sequencing was performed using an ABI3730XL Genetic Analyzer (Applied Biosystems; Thermo Fisher Scientific, Inc., Waltham, MA, USA) to detect mutations in the entire coding region of LICAM. Fetus sequence data was aligned with the reference sequence obtained from the University of California, Santa Cruz (Santa Cruz, CA, USA) Genome Browser (https://genome.ucsc.edu/; L1CAM, GRCh37/hg19, NM_000425.4) using Mutation Surveyor software (https://softgenetics.com/mutationSurveyor. php; version 4.0.4.0). Variants detected in the fetuses, but absent in the ClinVar (www.ncbi.nlm.nih.gov/clinvar/), Human Gene Mutation Database (www.hgmd.cf.ac. uk/ac/), L1CAM Mutation Database (http://www.11cammutationdatabase.info/mutations.aspx), Single Nucleotide
Polymorphism Database (www.ncbi.nlm.nih.gov/SNP), Ensembl (http://grch37.ensembl.org/index.html) and Exome Aggregation Consortium (ExAC; http://exac.broadinstitute. org/) were considered novel variants, and were further examined in the DNA samples of 100 healthy individuals, to exclude the possibility of ethnicity-specific polymorphisms.

In silico prediction and pathogenicity assessment. The amino acid sequences of human neural cell adhesion molecule L1CAM, obtained from the UniProt database (http://www.uniprot.org/), were aligned with orthologs from 6 different eukaryotic species by DNAMAN software (https://www.lynnon.com/; version 8.0), including Mus musculus, Bos taurus, Gallus gallus, Danio rerio, Xenopus tropicalis and Drosophila melanogaster. The pathogenicity of the identified variants was assessed using SIFT (http://sift.jcvi.org/), PolyPhen2 (http://genetics.bwh. harvard.edu/pph2/), and MutationTaster (http://www.mutationtaster.org/) prediction tools, and further classified following the guidelines of American College of Medical Genetics and Genomics (ACMG) /the Association for Molecular Pathology (AMP) (7).

Chromosome analysis revealed a normal male karyotype for the two fetuses, and molecular analysis identified two novel missense variants in $L 1 C A M$ from two families: c.998C $>$ T(p.Pro333Leu) in family 1 , and c. $2362 \mathrm{G}>\mathrm{T}$ (p.Val788Phe) in family 2 (Fig. 2). The affected fetuses were hemizygous, and the mothers of the fetuses were heterozygous; fathers were wild type for the identified variants. The older sister of family 2 was heterozygous and the younger sister was wild type. The proline residue at position 333 and the valine residue at position 788 of L1CAM are highly conserved across 7 eukaryotic species. The two variants were predicted to be 'disease causing' based on SIFT, PolyPhen2 and MutationTaster prediction tools. The residues were absent in the dbSNP, Ensembl, ExAC and the 100 normal controls. Alterations in amino acids at position 333 (Fig. 3A) had been reported as pathogenic in patients with hydrocephaly, and so, according to the ACMG/AMP guidelines, PM1, PM2 and PM5, in addition to PP3 and PP4, apply to this variant and is classified as likely pathogenic. The variant at position 788 (Fig. 3B), PM1, PM2, PP3 and PP4 also apply, and can also be classified as likely pathogenic.

\section{Discussion}

Congenital hydrocephaly is a common medical condition with complex and multifactorial causes. L1 syndrome accounts for $-5-10 \%$ of cases of congenital hydrocephaly in males. Molecular testing of LICAM for fetuses with isolated hydrocephaly may have limited usefulness (6). Additional clinical signs and family history can improve the diagnostic yield significantly (8). In the present study, it was demonstrated that $L 1 C A M$ testing for isolated hydrocephaly in a fetus with family history could lead to confirmatory molecular findings. Prior to the present study, there were only two L1CAM variants reported in Chinese patients (fetuses). The findings of the present study expand the mutational spectrum of the LICAM gene in the Chinese population, and the information can be used in genetic counseling, carrier testing of female relatives and prenatal, as well as preimplantation genetic diagnosis. 
Table I. Polymerase chain reaction primers for molecular analysis of the L1CAM gene.

Amplified product

Exon $\quad$ Forward $\left(5^{\prime}-3^{\prime}\right)$

Reverse (5'-3') size (bp)

\begin{tabular}{lllr}
\hline 1 & GTCCTTCCGTTCTCTCGTCT & AACGTCCTGGCTATCTCCTG & 397 \\
$2-4$ & AGCTTACTATGTCCCCTGCC & ATGAGATGACCGGAAGTGCA & 1401 \\
$5-6$ & CTGGCCTCCCTAAGTGCTAG & GCCTCTGTGTCTTCCTCCAT & 794 \\
$7-10$ & AATTCTGGGGTGGAGGGAAG & AGGGTGTCAGCAAGGAGAAA & 1178 \\
$11-15$ & AACCAAGATTTGCAGGGCTC & CCGACAATGGAGTGATCAGC & 1500 \\
$16-18$ & GCTGATCACTCCATTGTCGG & AATGCTGAGAGGTGTGGACA & 1020 \\
$19-23$ & TCTCTGTGTGTAGGGGCTTG & ACATACTGTGGCGAAAGGGA & 1461 \\
$24-27$ & TCCCTTTCGCCACAGTATGT & TGTTGGCCTCTCCCTGAAAT & 1250 \\
28 & AGATGACAGCTCCAGACCTG & AAGTTCTCCTCTGCCCCAAC & 494 \\
\hline
\end{tabular}

bp, base pair; L1CAM, L1 cell adhesion molecule.

A

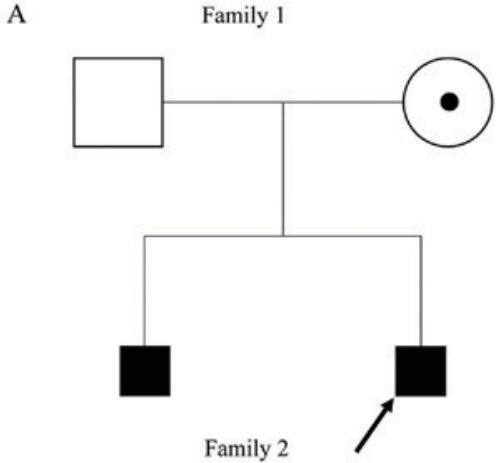

C

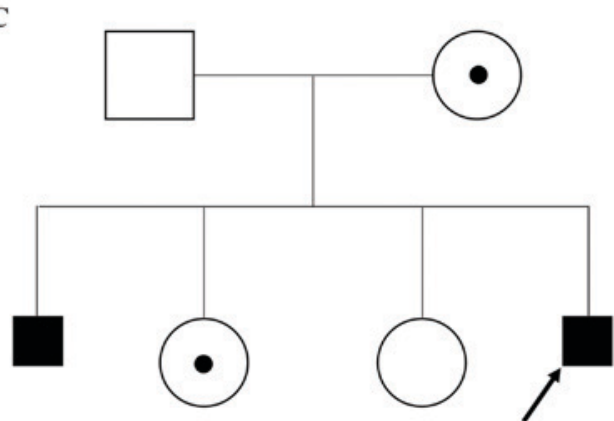

B

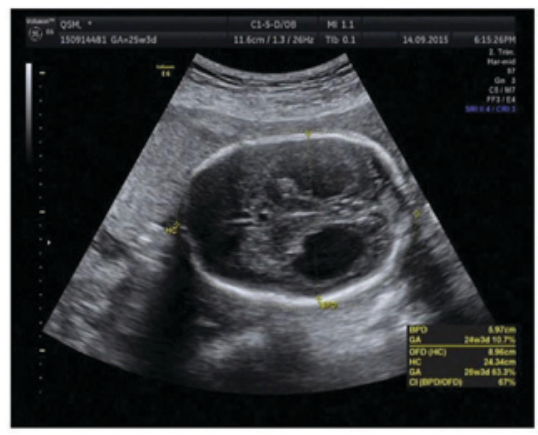

D

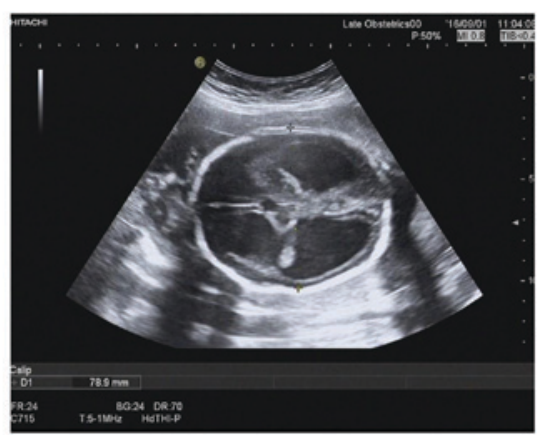

Figure 1. Family pedigrees with X-linked hydrocephalus and sonographic image for affected fetuses. (A) Pedigree of family 1 ; (B) severe hydrocephaly in fetus 1 as presented by ultrasonography. (C) Pedigree of family 2; (D) ultrasonography view of fetus 2 with severe hydrocephaly.

HSAS exhibits a variable phenotype within and between families. Female carriers exhibit no evident clinical symptoms or signs. The milder phenotype is characterized by low-grade hydrocephalus, whereas the severe phenotype is characterized by stillbirth or infant mortality (9). The L1CAM protein consists of six immunoglobulin (Ig) and five fibronectin type III (FnIII) domains in the extracellular region, a transmembrane domain and a short cytoplasmic $\mathrm{C}$ terminal domain (Fig. 3C) (10). Mutations occurring in the key residues are mostly considered to be pathogenic. Missense mutations in the extracellular domains may affect the integrity of the domains and disturb the binding activities of L1CAM to itself (homophilic) and to the other cell adhesion molecules including integrins and neurocan, causing mild or severe phenotypes, whereas mutations occurring in the cytoplasmic domain tend to exhibit the least severe effects, since the extracellular transmembrane domains remain intact (11).

Fetus 1 possessed a missense variant (c.998C $>$ T; p.Pro333Leu) impairing the fourth Ig domain of the extracellular domains. Proline 333 is a key residue that is buried in a close packed cavity and is important for the conformation of L1CAM (12). As a result of replacing an internal residue by a larger charged residue, the change of a proline to leucine likely results in the disruption of steric strain, thereby possibly reducing homophilic binding. This variant belongs to the class II of mutations, according to the classification 
Family 1

L1CAM: NM_000425.3

c. $998 \mathrm{C}>$ T (p. Pro333Leu)

A a Wild-type

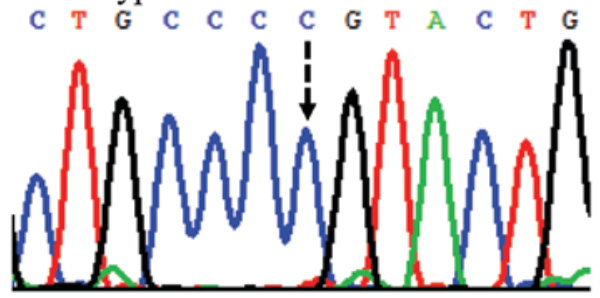

B a Heterozygous carrier

$\begin{array}{llllllllllllll}\text { C } & T & G & C & C & C & T & G & T & A & C & T & G\end{array}$

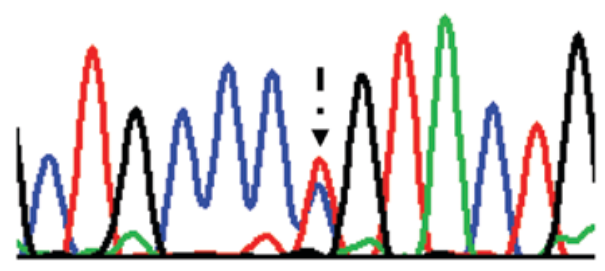

C a Hemizygous mutant

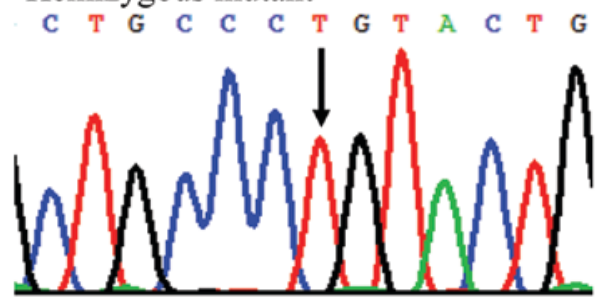

Family 2

L1CAM: NM_000425.3

c. $2362 \mathrm{G}>\mathrm{T}$ (p. Val788Phe)

A b Wild-type

$\begin{array}{lllllllllllll}\text { A } & \text { T } & \text { C } & \text { A } & \text { A } & \text { A } & G & \text { T } & \text { C } & \text { C } & \text { A } & \text { G } & \text { G }\end{array}$

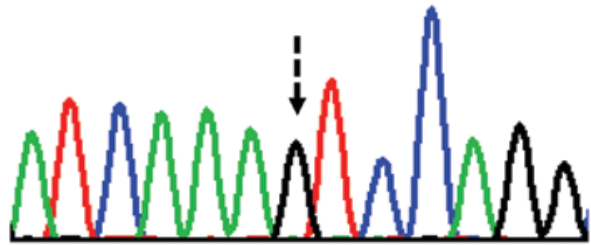

$\mathrm{B} \mathrm{b}$ Heterozygous carrier

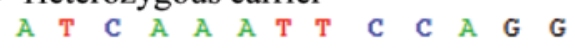

$\mathrm{C} \mathrm{b}$ Hemizygous mutant

$\begin{array}{lllllllllllllllllll}\text { A. } & \text { T } & \text { C } & \text { A. } & \text { A } & \text { A. } & \text { T } & \text { T } & \text { C } & \text { C } & \text { A. } & \text { G } & \text { G }\end{array}$

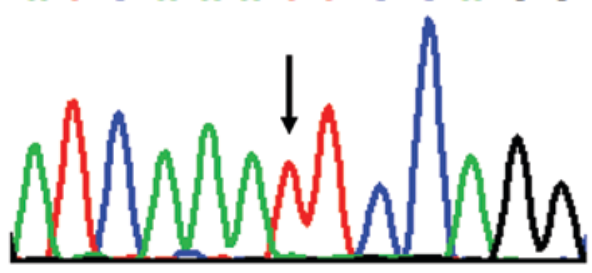

Figure 2. Partial DNA sequences in L1CAM of two families. (Aa and b) Wild type, (Ba and b) the heterozygous carrier forms. (Ca) Hemizygous c.998C $>\mathrm{T}$ (p.Pro333Leu) and (Cb) hemizygous c.2362G>T (p.Val788Phe) are identified in fetus 1 (family 1) and fetus 2 (family 2), respectively. L1CAM, L1 cell adhesion molecule.

A

\begin{tabular}{|c|}
\hline p.Pro333 \\
\hline Homo sapiens IARHAYYVTVEAR PYWLHKECSHLYG \\
\hline Mus musculus ¿ARHAYYVTVEAA PYWL CKECSSHYG \\
\hline Bos taurus:DRHAYYVTVEAAEYWLHKEQCSHIYG \\
\hline Gallus gallus 'ARGTHSVTVEAA FYWVRRECSGVFG \\
\hline Danio rerio ISTHTYRVTVEAA EYWIREERSCLYA \\
\hline $\begin{array}{l}\text { pus tropicalis SSHTFTVVVESAPYWINKEEDGIYA } \\
\text { melanogaster OSESITINVNSVYYFTRDEETATAA }\end{array}$ \\
\hline
\end{tabular}

B

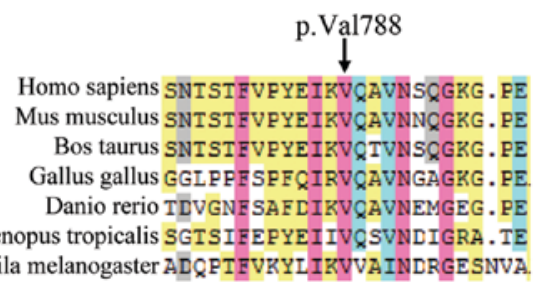

$\mathrm{C}$

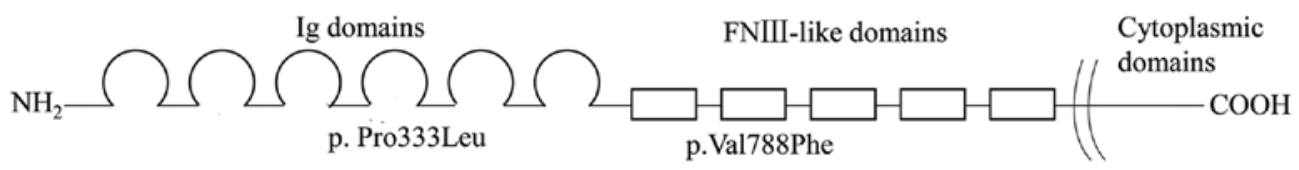

Figure 3. Multispecies alignment of L1CAM sequence and protein domain analysis. The multiple amino acids sequence alignment reveals that the (A) p.Pro333 and (B) p.Val788 residues are highly conserved during evolution. (C) Schematic representation of the L1CAM protein and two novel amino acid changes in relation to the domain structure of L1CAM protein. L1CAM, L1 cell adhesion molecule.

by Fransen et al (3). A different variant located in the same position (c.998C $>$ G; p.Pro333Arg) has been reported in a patient with hydrocephalus and adducted thumbs (13). Fetus 1 only demonstrated isolated hydrocephalus. The proline and leucine residues are hydrophobic uncharged amino acids, but arginine is a hydrophilic basic amino acid. The difference in the nature of the amino acids could lead to varying severity of the phenotype.

Fetus 2 possessed a missense class II variant (c. $2362 \mathrm{G}>\mathrm{T}$; p.Val788Phe) lying in the extracellular second FnIII domain. Valine 788 is involved in hydrogen bonding with another residue at 799-803. The introduction of a phenylalanine 
residue may disrupt the $\beta$-sheet hydrogen bonds and steric strain, affecting the structure and function of L1CAM protein.

In conclusion, the present study identified two novel pathogenic class II variants in L1CAM in two Chinese families with a history of hydrocephaly. The fetuses in these two families presented with isolated X-linked hydrocephaly, highlighting the importance of prenatal ultrasonography and LICAM mutation testing in the diagnosis of HSAS.

\section{Acknowledgements}

Not applicable.

\section{Funding}

The present study was supported by the project of science and technology of Guangxi Zhuang Autonomous Region (grant no. gui-ke-gong 14124004-1-8), and the Open Project Program of the Shanghai Key Laboratory of Birth Defect, Children's Hospital of Fudan University (grant no. 16DZKF1014).

\section{Availability of data and materials}

The data used and/or analyzed during the current study are available from the corresponding author or first author on reasonable request.

\section{Authors' contributions}

XF and BX designed the study and experiments. JL analyzed the clinical phenotype and followed up the patients. HW performed cordocentesis and induced abortion. ZY performed prenatal ultrasonography examination. SL and ZQ prepared reagents and designed the primers, QY and ML performed the PCR and Sanger sequencing experiments. SY and JW conducted the bioinformatic analysis. BX and YL made substantial contributions to the collation of data and wrote the manuscript. All authors read and approved the final manuscript.

\section{Ethics approval and consent to participate}

The present study was approved by Guangxi Maternal and Child Health Hospital Medical Ethics Committee [approval no. (2017) Lun Han Shen (3-10)] and written informed consent was obtained from parents and patients.

\section{Patient consent for publication}

Written informed consent was obtained from fetuses' parents for the publication of this study including images and data.

\section{Competing interests}

The authors declare that they have no competing interests.

\section{References}

1. Rosenthal A, Jouet M and Kenwrick S: Aberrant splicing of neural cell adhesion molecule L1 mRNA in a family with X-linked hydrocephalus. Nat Genet 2: 107-112, 1992.

2. Yamasaki M and Kanemura Y: Molecular biology of pediatric hydrocephalus and hydrocephalus-related diseases. Neurol Med Chir (Tokyo) 55: 640-646, 2015.

3. Fransen E, Van Camp G, D'Hooge R, Vits L and Willems PJ: Genotype-phenotype correlation in L1 associated diseases. J Med Genet 35: 399-404, 1998.

4. Muñoz A,Cabrera-López JC,Santana-Rodríguez A, Toledo-Bravo de Laguna L, Santana-Artiles A and Sebastián-García I: X-linked hereditary spastic paraplegia due to mutation in the L1CAM gene: Three cases reports of CRASH syndrome. Rev Neurol 62: 218-222, 2016 (In Spanish).

5. Yao F, Zhang W, Gao J, Chen W and Liu X: Detection of L1CAM mutation and prenatal diagnosis of X-linked hydrocephalus. Sheng Zhi Yi Xue Za Zhi 25: 347-352, 2016 (In Chinese).

6. Duan H, Zhao G, Wang Y, Zhu X and Li J: Novel missense mutation of L1CAM in a fetus with isolated hydrocephalus. Congenit Anom (Kyoto) 58: 176-177, 2018.

7. Richards S, Aziz N, Bale S, Bick D, Das S, Gastier-Foster J, Grody WW, Hegde M, Lyon E, Spector E, et al: Standards and guidelines for the interpretation of sequence variants: A joint consensus recommendation of the American college of medical genetics and genomics and the association for molecular pathology. Genet Med 17: 405-424, 2015.

8. Adle-Biassette H, Saugier-Veber P, Fallet-Bianco C, Delezoide AL, Razavi F, Drouot N, Bazin A, Beaufrère AM, Bessières $\mathrm{B}$, Blesson $\mathrm{S}$, et al: Neuropathological review of 138 cases genetically tested for X-linked hydrocephalus: Evidence for closely related clinical entities of unknown molecular bases. Acta Neuropathol 126: 427-442, 2013.

9. Chidsey BA, Baldwin EE, Toydemir R, Ahles L, Hanson H and Stevenson DA: L1CAM whole gene deletion in a child with L1 syndrome. Am J Med Genet A 164A: 1555-1558, 2014.

10. Bateman A, Jouet M, MacFarlane J, Du JS, Kenwrick S and Chothia C: Outline structure of the human L1 cell adhesion molecule and the sites where mutations cause neurological disorders. EMBO J 15: 6050-6059, 1996.

11. Shieh C, Moser F, Graham JM Jr, Watiker V and Pierson TM: Mutation in the sixth immunoglobulin domain of L1CAM is associated with migrational brain anomalies. Neurol Genet 1: e34, 2015.

12. De Angelis E, Watkins A, Schäfer M, Brümmendorf T and Kenwrick S: Disease-associated mutations in L1 CAM interfere with ligand interactions and cell-surface expression. Hum Mol Genet 11: 1-12, 2002.

13. De Angelis E, MacFarlane J, Du JS, Yeo G, Hicks R, Rathjen FG, Kenwrick $S$ and Brümmendorf T: Pathological missense mutations of neural cell adhesion molecule L1 affect homophilic and heterophilic binding activities. EMBO J 18: 4744-4753, 1999. 